
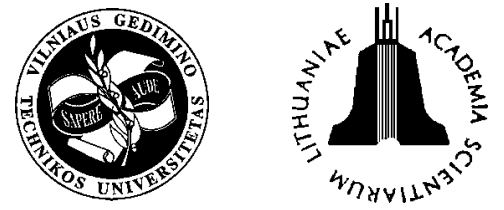

ISSN 1392-3730

JOURNAL OF CIVIL ENGINEERING AND MANAGEMENT

http:/www.vtu.lt/english/editions

2004, Vol X, No 3, 217-226

\title{
TRUSS OPTIMIZATION UNDER COMPLEX CONSTRAINTS AND RANDOM LOADING
}

\author{
Arnoldas Norkus ${ }^{1}$, Romanas Karkauskas ${ }^{2}$ \\ Vilnius Gediminas Technical University, Saulètekio al. 11, LT-10223 Vilnius-40, Lithuania. \\ E-mail:1 arnoldas.norkus@st.vtu.lt; 2 rokark@st.vtu.lt
}

Received 2 Feb 2004; accepted 17 Apr 2004

\begin{abstract}
An actual design of light-weight structures must evaluate strength, stiffness and stability constraints as well as the nature of external loading. A designed structure must satisfy optimality and safety criterions per prescribed maintenance period. One faces the known difficulties when trying to implement several from the above-mentioned requirements into optimization problem for further successful numerical realisation. A method to formulate the optimization problem, incorporating all above described criterions, the mathematical model and algorithm to solve it numerically, taking into account the stochastic nature of external loading, are presented for elastic-plastic truss-type structure.
\end{abstract}

Keywords: elastic-plastic structure; optimization; stiffness and stability constraints; stochastic loading process, finite element discrete model.

\section{Introduction}

The aim of structural design is a creation of a structure to satisfy optimality and safety criterions in respect of maintenance (strength, stiffness, stability) requirements. It is important to note that stiffness and stability constraints often dominate versus strength conditions in actual design of optimal structures. When solving the limit equilibrium problem [1-3], the structural parts deformations or nodal displacements can exceed the fixed admissible magnitudes and/or fail due to stability loss. Actually, the structure is designed for a certain period for prescribed reliability level. Therefore one must also seek to evaluate the loading process peculiarities (periods of activity and absence of separate loads when combining their common influence on structural response per reference period of time) in the practical design. An employment of elastic-plastic structural behaviour model allows to evaluate the residual response effects. The appeared self-equilibrated internal forces allow to adapt the prescribed reliability level optimal structure to variable, random in general, process of external loading [4]. Such an approach allows to evaluate the alternating plasticity case and thus the structure fail mode due to low-cycle fatigue. This failure case is the actual one in structural analysis and design $[5,6]$.

Thus, the main optimal structural design problem is to be stated as the structural optimization problem under presence of stiffness, stability requirements and generally, the stochastic process of loading.

The designed optimal structure is in the state prior to plastic failure, resulting from stiffness and stability constraints. During the optimization procedures the trusstype discrete structure tensile members are assumed to be loaded up to the yield limit, the compressive members - up to the critical limits, combining yielding and buckling modes, the stiffness constraints are represented via the nodal displacements restrictions in prescribed directions. The problem solution realises the certain optimization cycles, in each cycle employing the set of members limit forces, identified in the previous one. The optimization cycles are continued until a certain convergence.

The structure limit states represent strength, stiffness and stability loss failure modes. The total structure failure probability $P_{r f}$ is a union of the above modes. Taking the structure physical and geometrical properties to be described as random values, the structure $P_{r f}$ evaluation (reliability analysis) problem is rather complicated even applying the constant random loads and evaluating strength conditions only (ie limit states correspond to different plastic collapse mechanisms modes) [7, 8]. The reliability evaluation problem, stated as the mathematical non-convex mathematical programming problem, solution process results in the selection of the most reliable plastic mechanisms, corresponding to local extremums, in order to calculate $P_{r f}$ versus plastic collapse (note that different plastic mechanisms can be selected in each optimization cycle). The number of plastic mechanisms (failure modes) increases considerably with the number of redundancy.

An additional evaluation of displacement limitations, that of stability loss limit states in concert with stochas- 
tic process of loading makes the main optimal structural design problem rather complicated even for the elementary structures and is more interesting in theoretical versus practical usage.

Let the physical and geometrical properties of structural members and the loading application points represent as determinate ones. The actual variation of carrying structural members properties in principle must be low, therefore under certain restrictions can be taken as determinate ones, fixed with sufficiently high reliability level. Then the actual elastic response of elastic-plastic structure (in terms of internal forces and displacements), subjected by stochastic loading process can be stated as the above extreme (due to certain reliability per reference period of time) values identification problem. After when these identified values are fixed as determinate ones, the main optimization problem of the structure in terms of determinate values is to be solved. The similar approach, taking into account only strength conditions, was applied [4].

A direct application of the mathematical programming method [9] for optimization problem solution does not ensure the obtained extremum to be the global one. The way to overcome the latter lack is an application of mathematical programming theory in concert with extreme energy principles. The obtained optimization problem, containing the complementarity condition, is the multi-extremum one, as its solution results in a certain number of discrete points. Incorporation of the complementarity's condition into the objective function of optimization problem [10-12] ensures the admissible set to become the convex one in case of convex yield conditions, having the unique optimal solution. But one must note that problem numerical solution via mathematical programming methods is very complicated and in some cases unavailable.

The task of the present investigation is further development of the reliability-based optimization under presence stiffness and stability constraints problem solution methods, applying the extreme energy principle [2, 12] in concert with certain optimization procedures for elastic-plastic truss-type structure (eg truss), subjected by stochastic process of loading.

\section{Truss optimization problem mathematical model and algorithm}

The truss optimization problem under the presence of stiffness, stability constraints and stochastic process of loading consists of 3 principal parts of optimization cycles (iterations):

1. Determining truss variation bounds of elastic forces $\mathbf{S}_{e}^{+}, \mathbf{S}_{e}^{-}$and displacements $\mathbf{u}_{e}^{+}, \mathbf{u}_{e}^{-}$per time period $T$, satisfying prescribed safety reliability $P_{r s}=1-P_{r f}$.

2. Defining the actual stress and strain state (SSS) of the structure, prior to plastic collapse, correspond- ing to fixed variation bounds of internal forces and displacements (analysis problem).

3. Optimizing the areas of members (conditioning the limit $\mathbf{S}_{0}$ and that of critical $\mathbf{S}_{c r}$ forces) to satisfy strength, stiffness, stability and constructional constraints.

Each of the parts consists of separate problems, to be solved individually one after another during each optimization cycle and continued until certain convergence. The iterative optimization procedure is conditioned by the circumstance that elastic forces and displacements from one side and limit and critical forces from the other side depend on the actual cross-sectional areas of bars, being as input and output data of optimization cycle parts 1 and 3.

\subsection{Evaluation of elastic forces and displacements caused by stochastic loading process}

Let the loading process to be described by action of individual loads $\tilde{F}(t) \equiv\left(\tilde{F}_{1}(t), \tilde{F}_{2}(t), \ldots, \tilde{F}_{m}(t)\right)^{T}$. Then the $j$-th elastic axial force and the $i$-th elastic displacement of the truss are expressed, respectively, by:

$$
\begin{aligned}
& \tilde{S}_{e, j}(t)=\tilde{S}_{e 1, j}(t)+\tilde{S}_{e 2, j}(t)+\ldots+\tilde{S}_{e m, j}(t)= \\
& \gamma_{j 1} \cdot \tilde{F}_{1}(t)+\gamma_{j 2} \cdot \tilde{F}_{2}(t)+\ldots+\gamma_{j m} \cdot \tilde{F}_{m}(t)= \\
& \sum_{i=1}^{m} \gamma_{j i} \cdot \tilde{F}_{i}(t), \quad j=1, \ldots, n, \\
& \tilde{u}_{e, i}(t)=\tilde{u}_{e l, i}(t)+\tilde{u}_{e 2, i}(t)+\ldots+\tilde{u}_{e m, i}(t)= \\
& \vartheta_{i 1} \cdot \tilde{F}_{1}(t)+\vartheta_{i 2} \cdot \tilde{F}_{2}(t)+\ldots+\vartheta_{i m} \cdot \tilde{F}_{m}(t)= \\
& \sum_{i=1}^{m} \vartheta_{i i} \cdot \tilde{F}_{i}(t),
\end{aligned}
$$

where $\gamma_{j m}$ and $\vartheta_{i m}$ are the truss finite element method discrete model $j$-th elastic axial force and $i$-th elastic displacement influence determinate coefficients versus $m$-th load process $\tilde{F}_{m}(t)$, respectively.

To identify the maximum and the minimum magnitudes of processes (1) per reference period of time $T$ due to prescribed design safety reliability $P_{r d}=P_{r s}=1-P_{r f}$ for all internal forces, selected into the vectors

$$
\begin{gathered}
\mathbf{S}_{e}^{+}=\left(S_{e, j}^{+}\right)^{T} \equiv\left(S_{e, 1}^{+}, S_{e, 2}^{+}, \ldots, S_{e, n}^{+}\right)^{T}, \\
\mathbf{S}_{\mathrm{e}}^{-}=\left(S_{e, j}^{-}\right)^{T} \equiv\left(S_{e, 1}^{-}, S_{e, 2}^{-}, \ldots, S_{e, n}^{-}\right)^{T},
\end{gathered}
$$

and that of nodal displacements

$$
\begin{gathered}
\mathbf{u}_{e}^{+}=\left(u_{e, i}^{+}\right)^{T} \equiv\left(u_{e, 1}^{+}, u_{e, 2}^{+}, \ldots, u_{e, m}^{+}\right)^{T}, \\
\mathbf{u}_{e}^{-}=\left(u_{e, i}^{-}\right)^{T} \equiv\left(u_{e, 1}^{-}, u_{e, 2}^{-}, \ldots, u_{e, m}^{-}\right)^{T},
\end{gathered}
$$


one must solve the following problem:

$$
\begin{aligned}
& \mathrm{P}_{\mathrm{r}}\left(\begin{array}{l}
\mathbf{S}_{\mathrm{e}}^{+} \leq \tilde{\mathbf{S}}(t) \leq \mathbf{S}_{\mathrm{e}}^{-} ; \\
\mathbf{u}_{\mathrm{e}}^{+} \leq \tilde{\mathbf{u}}(t) \leq \mathbf{u}_{\mathrm{e}}^{-}
\end{array}\right)=P_{r d}, \\
& t=[0, T] .
\end{aligned}
$$

An application of the exact methods (eg random crossing theory) for processes combination (Eqn (1)) problem seems to be rather complicated one, dealing with multiple convolution integrals calculation problems to build the above values distribution and density functions. The latter are required for elastic response extreme magnitudes identification due to fixed design probability $P_{r d}[13,14]$. The number of such processes, to be investigated, is equal to $n+m$, ie to the total number of components of internal forces and displacement vectors (2). The number of these operations (recalculations of the above-mentioned elastic response magnitudes, provided in the first part of each optimization cycle), also depends on the successful problem solution starting point, chosen for faster convergence of the main optimization problem.

Thus, the exact evaluation methods of extreme elastic response values seems to be more interesting from the theoretical point of view, being in contradiction when applying them for practical usage to solve real main structural optimization problems.

Taking into account peculiarities of the investigated problem, the loading process approximation (load combination) problem useful approach would be the selection of process finite number time points, to define the extreme influence of loading to structure elastic response values. The Turkstra's load combination rule, applied for mutually independent load processes, proposes the number of such points to be equal to the number of individual loads processes. The employed rule, being implemented in design codes of several countries, provides sufficient lower bound probability evaluation to identify the extreme magnitudes of considered values. But one must note that the Turkstra's rule accuracy is also limited by certain features of loads processes to be combined [15].

Following the Turkstra's rule idea to solve the problem (1) numerically, the aim is to represent the loading process by certain number of individual loads determinate magnitudes combinations, sufficient to identify the extreme loading influence to all structural elastic response values. The identified combinations of individual loads in terms of determinate magnitudes must be selected to the loading matrix (matrixes), being constant during all iterative optimization procedures. The loading matrices represent the possible loading states - combinations of individual loads magnitudes, corresponding to prescribed reliability level and time period under consideration.

Let all the individual loads be mutually independent, stationary processes. Applying the Borges-
Castanheta load model, the load process is approximated as a sequence of rectangular pulses of fixed duration $\tau$, taking into account probability of load absence during the pulse. Then the load distribution function for pulse reads:

$$
P_{F, \tau}=\left\{\begin{array}{r}
p^{*}+q^{*} \hat{P}_{F}(F), \text { when } F>0, \\
q^{*} \hat{P}_{F}(F), \text { when } F<0, \\
p^{*}+q^{*}=1,
\end{array}\right.
$$

where $\hat{P}_{F}(F)$ is the load distribution function during its activity period; $p^{*}$ - the load absence probability during pulse $\tau$.

The load magnitude, corresponding to certain probability $P_{F, \tau}$ is found from Eqn (3), taking into account the load absence probability, ie:

$$
P_{F, \tau}=\left\{\begin{array}{l}
\hat{P}_{F}^{-1}\left(\frac{P_{F, \tau}(F)}{q^{*}}\right) \text { when } 0 \leq P_{F, \tau}(F) \leq q^{*} \hat{P}_{F}(0), \\
0, \text { when } q^{*} \hat{P}_{F}(0) \leq P_{F, \tau}(F) \leq p^{*}+q^{*} \hat{P}_{F}(0), \\
\hat{P}_{F}^{-I}\left(\frac{P_{F, \tau}(F)-p^{*}}{q^{*}}\right), \text { when } p^{*}+q^{*} \hat{P}_{F}(0) \leq \\
\leq P_{F, \tau}(F) \leq 1 .
\end{array}\right.
$$

Then the load extreme magnitudes distribution functions per reference period $T$ read:

$$
\begin{aligned}
& P_{\max , F}(F)=\left(P_{F, \tau}(F)\right)^{v}, \quad P_{\min x, F}(F)=1-\left(1-\left(P_{F, \tau}(F)\right)\right)^{v}, \\
& v=T / \tau .
\end{aligned}
$$

The Turkstra's rule does not limit the number of loads when combining their extreme effect, but usually it is enough to evaluate three time-variable forces (when required, the preliminary reliability investigation of common activity periods of more variable loads must be provided). Then the extreme, for instance, internal force (Eqn (1)) magnitude with required $P_{r d}$ per reference period $T$, is achieved, analysing the following loading process time points:

$$
\begin{aligned}
& P_{\text {ekstr } \sum_{i=1}^{3}(S e i)}\left(P_{r d, e k s t r}\right) \approx \\
& \left\{\begin{array}{l}
P_{e k s t r S_{e l, T}}^{-1}\left(P_{r d, e k s t r}\right)+P_{e k s t r S_{e l, \tau l}}^{-1}(0,5)+P_{e k s t r S_{e l, \tau l}}^{-1}(0,5), \\
P_{e k s t r S_{e l, T}}^{-1}\left(P_{r d, e k s t r}\right)+P_{e k s t r S_{e l, \tau l}}^{-1}(0,5)+P_{e k s t r S_{e l, \tau l}}^{-1}(0,5), \\
P_{e k s t r S_{e l, \tau l}}^{-1}(0,5)+P_{e k s t r S_{e l, T}}^{-1}\left(P_{r d, e k s t r}\right)+P_{e k s t r S_{e l, \tau l}}^{-1}(0,5), \\
P_{e k s t r S_{e l, \tau l}}^{-1}(0,5)+P_{e k s t r S_{e l, \tau l}}^{-1}(0,5)+P_{e k s t r S_{e l, T}}^{-1}\left(P_{r d, e k s t r}\right),
\end{array}\right.
\end{aligned}
$$

where $\tau_{1}>\tau_{2}>\tau_{3}$.

When calculating determinate maximum and minimum magnitudes, it is proposed to select the quintiles by:

$$
P_{r d}=P_{r d(\max )}-P_{r d(\min )}
$$


and 0,5 , as it fit sufficiently with magnitudes, identified by exact, random crossing methods $[16,17]$.

When applying the described loading process approximation method to evaluate all extreme elastic response values, the loading matrices, selecting the required loads determinate magnitudes combinations, (eventual loading process extreme states) are:

$$
\left[F^{+}\right]=\left[\begin{array}{cccc}
\max _{T} F_{1}(t) & \max _{T} F_{1}(t) & F_{1}(t) & F_{1}(t) \\
\max _{\tau_{1}} F_{2}(t) & F_{2}(t) & \max _{T} F_{2}(t) & F_{2}(t) \\
\max _{\tau_{2}} F_{3}(t) & \max _{\tau_{1}} F_{3}(t) & \max _{\tau_{2}} F_{3}(t) & \max _{T} F_{3}(t)
\end{array}\right],
$$

$$
\left[F^{-}\right]=\left[\begin{array}{cccc}
\min _{T} F_{1}(t) & \min _{T} F_{1}(t) & F_{1}(t) & F_{1}(t) \\
\min _{\tau_{1}} F_{2}(t) & F_{2}(t) & \min _{T} F_{2}(t) & F_{2}(t) \\
\min _{\tau_{2}} F_{3}(t) & \min _{\tau_{1}} F_{3}(t) & \min _{\tau_{2}} F_{3}(t) & \min F_{3}(t)
\end{array}\right],
$$

where determinate values correspond to extreme $P_{r d(\max )}$, $P_{r d(\min )}($ Eqn (7)) and 0,5 quantiles.

Note, that taking into account Eqns (4) and (5), the extreme magnitudes per considered period of time are calculated by improved design probabilities:

$$
\begin{gathered}
\max _{T, \tau i} F_{i}(t)=F_{F}^{+}=\hat{P}_{F}^{-1}\left(\frac{\sqrt[v]{P_{r(\max )}}-p^{*}}{q^{*}}\right)=\hat{P}_{F}^{-1}\left(P_{r(\max )}^{*}\right), \\
\min _{T, \tau i} F_{i}(t)=F_{F}^{-}=\hat{P}_{F}^{-1}\left(\frac{1-\sqrt[v]{1-P_{r(\min )}}}{q^{*}}\right)=\hat{P}_{F}^{-1}\left(P_{r(\min )}^{*}\right), \\
v=\frac{T}{\tau_{i}} \text { or } \frac{\tau_{i}}{\tau_{j}} \text { for } \tau_{i}>\tau_{j}, \quad j=1,2,3 .
\end{gathered}
$$

Find that the quantiles, resulting the different sign extreme magnitudes of loads (Fig 1), are constrained by

$$
\begin{aligned}
& P_{r(\max )}^{*}>\left(p^{*}+q^{*} \hat{P}_{F}(0)\right)^{v} \text { for } \max _{i}(t)>0, \\
& \text { and } P_{r(\text { min })}^{*}<\left(1-\left(1-q^{*} \hat{P}_{F}(0)\right)^{v} \text { for } \min _{i}(t)>0 .\right.
\end{aligned}
$$

The vectors of extreme elastic response magnitudes then are formed from obtained elastic solution in terms of matrices

$$
\begin{aligned}
& {\left[S_{e}^{+}\right]=\left\lfloor\gamma^{+}\right] \cdot\left[F^{+}\right]+\left\lfloor\gamma^{-}\right] \cdot\left[F^{-}\right],} \\
& {\left[S_{e}^{-}\right]=\left[\gamma^{+}\right] \cdot\left[F^{-}\right]+\left[\gamma^{-}\right],} \\
& {\left[u_{e}^{+}\right]=\left[\vartheta^{+}\right] \cdot\left[F^{+}\right]+\left[\vartheta^{-}\right] \cdot\left[F^{-}\right],} \\
& {\left[u_{e}^{-}\right]=\left[\vartheta^{+}\right] \cdot\left[F^{-}\right]+\left[\vartheta^{-}\right]}
\end{aligned}
$$

by selecting certain extreme magnitudes from corresponding matrices lines.

The above elastic response influence matrices are split to the ones, consisting of positive and zero or negative and zero magnitudes [2], to satisfy:

$$
[\gamma]=\left[\gamma^{+}\right]+\left[\gamma^{-}\right] \text {and }[\vartheta]=\left[\vartheta^{+}\right]+\left[\vartheta^{-}\right] \text {. }
$$

Note, that matrices (8) in coordinate space of individual loads represent the convex polyhedron (created according to fixed reliability $P_{r d}$ ) outer surface apexes, corresponding to stochastic loads possible common action states. An assemblage of these apexes to matrix $[F]$ provides all possible extreme structural response causing loading cases:

$$
\left[S_{e}\right]=[\gamma] \cdot[F] \text { and }\left[u_{e}\right]=[\vartheta] \cdot[F] .
$$

Then the extreme elastic response magnitude vectors are formed by selecting certain extreme magnitudes from corresponding above matrices lines. This approach versus to that of Eqn (11) requires more efforts when selecting all apexes and is applicable for less number of individual forces cases, when their action is prespecified by non-alternating direction processes, other cases.

\subsection{Mathematical model of analysis problem}

The structure is subjected to the known external loading, the areas and physical-geometrical properties of structural members are fixed. The structure, prior to plastic collapse, SSS evaluation problem is realised by the following mathematical model $[12,18]$ for discrete trusstype structure finite element model:

find

$-\frac{1}{2} \lambda_{1}^{T}[G] \lambda_{1}+\lambda_{1}^{T}[G] \lambda_{2}-\frac{1}{2} \lambda_{2}^{T}[G] \lambda_{2}+\lambda_{1}^{T}\left(\mathbf{S}_{e}^{+}-\mathbf{S}_{0}\right)+$ $\lambda_{2}^{T}\left(-\mathbf{S}_{e}^{-}-\mathbf{S}_{c r}\right) \rightarrow \max$

subject to

$$
\boldsymbol{\lambda}_{1} \geq \mathbf{0}, \quad \boldsymbol{\lambda}_{2} \geq \mathbf{0}
$$

The quadratic programming problem (14) contains the following values:

$\mathbf{S}_{e}^{+}=\left(S_{e, j}^{+}\right)^{T}=\left(S_{e, 1}^{+}, S_{e, 2}^{+}, \ldots, S_{e, n}^{+}\right)-$vector of structural extreme positive axial forces; 


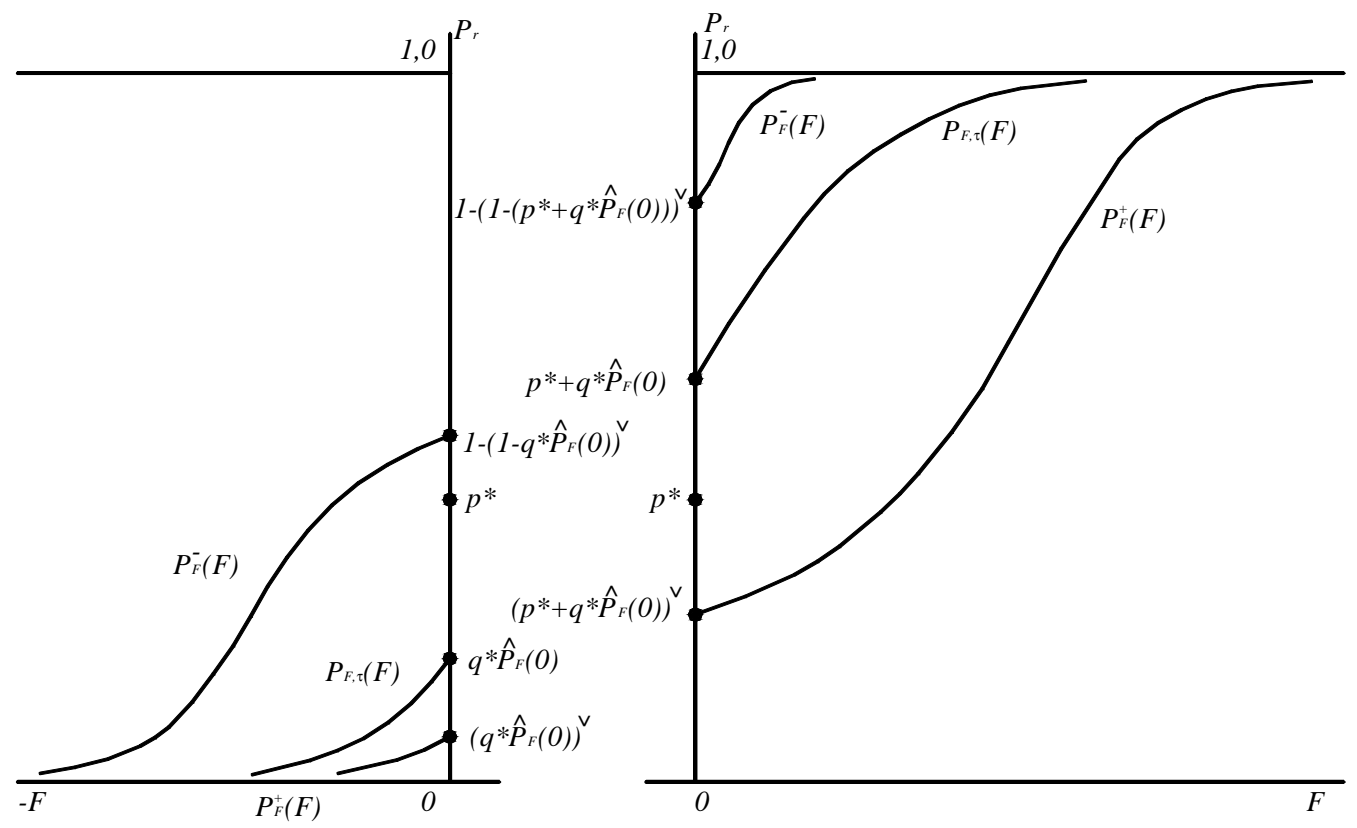

Fig 1. Distribution functions for extreme load magnitudes

$\mathbf{S}_{e}^{-}=\left(S_{e, j}^{-}\right)^{T}=\left(S_{e, 1}^{-}, S_{e, 2}^{-}, \ldots, S_{e, n}^{-}\right)-$vector of structural extreme negative axial forces;

$\lambda_{1}=\left(\lambda_{1, j}\right)^{T}-$ vector of Lagrange multipliers of complementarity conditions for tensile bars, reading

$$
\lambda_{1, j}\left(S_{r, j}+S_{e, j}^{+}-S_{0}\right)=0 ;
$$

$S_{r, j}$ - the $j$-th finite element (bar) residual force in the running optimization cycle; $S_{0, j}-j$-th finite element limit axial force (yielding force), $S_{0, j}=\sigma_{y} A_{j}, \sigma_{y}-$ the material yield limit, $A_{j}-j$-th finite element area, arbitrarily fixed or identified via previous optimization cycle; $\lambda_{2}=\left(\lambda_{2, j}\right)^{T}$ - the Lagrange multipliers of complementarity's conditions for compressive bars, reading

$$
\lambda_{2, j}\left(-S_{r, j}-S_{e, j}^{-}-S_{c r, j}\right)=0,
$$

where $S_{c r, j}-j$-th finite element limit buckling-yielding axial force, arbitrarily fixed or identified via previous optimization process; $[G]$ - constant per optimization process structure finite element discrete model $(n \times n)$ dimensional influence matrix for residual internal forces, reading

$$
[G]=\left[[B][\bar{K}][R][K]^{-1}[R]^{T}[\bar{K}][B]^{T}-[K]\right] .
$$

Here:

$[K]$ - tension-compression diagonal stiffness $\frac{E A_{j}}{l_{j}}$ matrix of structural elements $j=1,2, \ldots, n((n-$ total number of bars; $E, l_{j}$ - the material elasticity modulus and the $j$-th bar length, respectively); $[K]-$ the structure finite element $(m \times m)$-dimensional discrete model global stiffness matrix, where $m$ - number of global displacements;
$[B]-(n \times 6 n)$-dimensional configuration matrix, containing either zero elements or configuration submatrices for internal forces

$$
\left[B_{j}\right]=\left(b_{j, k}\right), k=6 j-5,6 j-4, \ldots, 6 j,
$$

expressed via direction cosines in the global coordinate system:

$$
\left[B_{j}\right]=\left(\begin{array}{llllll}
0 & 0 & 0 & \cos \alpha_{j} & \cos \beta_{j} & \cos \gamma_{j}
\end{array}\right) ;
$$

$[\bar{K}]_{-}$quasidiagonal $(6 n \times 6 n)$-dimensional matrix, containing $j$-th space finite element stiffness diagonal submatrices

$$
\left[K_{j}\right]=\frac{E A_{j}}{l_{j}}\left[\begin{array}{rr}
{\left[\bar{k}_{j}\right]} & -\left[\bar{k}_{j}\right] \\
-\left[\bar{k}_{j}\right] & {\left[\bar{k}_{j}\right]}
\end{array}\right]
$$

in the global coordinate system, where:

$$
\left[\bar{k}_{j}\right]=\left[\begin{array}{lll}
\cos ^{2} \alpha_{j} & \cos \alpha_{j} \cos \beta_{j} & \cos \alpha_{j} \cos \gamma_{j} \\
\cos \beta_{j} \cos \alpha_{j} & \cos ^{2} \beta_{j} & \cos \beta_{j} \cos \gamma_{j} \\
\cos \gamma_{j} \cos \alpha_{j} & \cos \gamma_{j} \cos \beta_{j} & \cos ^{2} \gamma_{j}
\end{array}\right]
$$

$[R]-(6 n \times m)$ dimensional configuration matrix of local and global displacements, containing unit and zero components.

Note that introducing the above finite element discrete model notations, the internal force and nodal displacement influence coefficients $\gamma$ and $\vartheta$ in (1) are obtained from the matrices

$$
[v]=[G], \quad[\vartheta]=\left[[K]^{-1}[R]^{T}[\bar{K}][B]^{T}\right] .
$$


The solution of the quadratic programming problem (14) yields the Lagrange multipliers $\lambda_{1}$ and $\lambda_{2}$ magnitudes. The components of residual internal forces $\mathbf{S}_{\mathrm{r}}=\left(S_{r, j}\right)^{T} \equiv\left(S_{r, 1}^{-}, S_{r 2}^{-}, \ldots, S_{r, n}^{-}\right)^{T}$ and that of residual displacements $\mathbf{u}_{\mathrm{r}}=\left(u_{r, i}\right)^{T} \equiv\left(u_{r, 1}, u_{r, 2}, \ldots, u_{r, m}\right)^{T}$ are determined by

$$
\mathbf{S}_{\mathrm{r}}=[G]\left(\lambda_{1}-\lambda_{2}\right), \quad \mathbf{u}_{\mathrm{r}}=[\vartheta]\left(\lambda_{1}-\lambda_{2}\right)
$$

Note, that one cannot state the above obtained residual structural response values to be the actual ones. Generally, the exact distribution of residual values depends upon the loading history, observed/simulated from individual loads processes [19]. Actually, in case of stochastic loading, the loading history prediction practically is unavailable. Thus, an employment of mathematical model (14) yields only the possible distribution of residual structural response values, ensuring adaptation of optimal structure to most dangerous eventual stochastic loading states corresponding to prescribed reliability level per reference period of time.

Having identified the above-mentioned values of residual response, the extreme magnitudes of internal forces and displacements are obtained by

$$
\begin{array}{ll}
\mathbf{S}^{+}=\mathbf{S}_{r}+\mathbf{S}_{e}^{+}, & \mathbf{S}^{-}=-\mathbf{S}_{r}-\mathbf{S}_{e}^{-} \\
\mathbf{u}^{+}=\mathbf{u}_{r}+\mathbf{u}_{e}^{+}, & \mathbf{u}^{-}=-\mathbf{u}_{r}-\mathbf{u}_{e}^{-}
\end{array}
$$

\subsection{Mathematical optimization model of structure bars cross-sectional areas}

The structure bars cross-sectional areas optimization mathematical model under stiffness and stability constraints consists of:

1. Axial strength conditions expressed via areas of optimised bars;

2. Strength conditions versus buckling of bars;

3. Displacements limitations constraints;

4. Constructive limitations for bars areas.

The structure optimality criterion expresses total material minimum weight of the bars.

Thus, the structure optimization model under presence of all the above-mentioned conditions is as follows:

$$
\text { find } W=\rho \sum_{k=1}^{n_{0}} A_{k} \sum_{r=1}^{n_{k}} l_{r} \rightarrow \min
$$

\section{subject to}

$$
\begin{aligned}
& -\sigma_{y} A_{k, j} \leq-S_{j}^{+}, \\
& -\sigma_{c r} A_{k, j} \leq S_{j}^{-}, k=1,2, \ldots, n_{0}, \\
& u_{t}=\sum_{j=1}^{n} \frac{S_{t, j}^{\max } \cdot \bar{S}_{t, j} \cdot l_{j}}{E A_{k, j}} \leq u_{t, \text { max }}, \\
& u_{t}=\sum_{j=1}^{n} \frac{S_{t, j}^{\min } \cdot \bar{S}_{t, j} \cdot l_{j}}{E A_{k, j}} \geq u_{t, \text { min }}, t=1,2, \ldots, m_{t}, \\
& A_{k} \geq A_{k, \text { min }},
\end{aligned}
$$

where: $A_{k}$ is the optimized cross-sectional area of the $k$-th group of bars; $n_{k}$ is the number of bars in $k$-th group; $l_{r}$ is the total length of bars, corresponding to $k$-th group; $n_{1}+n_{2}+\ldots+n_{n_{0}}=n ; n_{0}$ is the number of optimized parameters - ie the number of different group of bars; $\rho$ is the structure material density; $\sigma_{y}, \sigma_{c r}$ are material yield and critical stresses for tensile and compressive-buckled members, respectively; $u_{t}$ is the displacement, being constrained along the certain direction $t ; m_{t}$ is the number of constrained displacements; $u_{t, \text { max }}>0, \quad u_{t, \text { min }}<0$ are the upper and the lower bounds for displacement magnitudes to be constrained; $A_{k, \min }$ is the lower bound of cross-sectional area $A_{k}$ magnitude (constructive limitation).

Applying the virtual displacement principle, the displacement $u_{t}$ can be expressed by

$$
u_{t}=\sum_{j=1}^{n} \frac{S_{t, j}^{e x t} \cdot \bar{S}_{t, j} \cdot l_{j}}{E A_{k, j}}
$$

where $S_{t, j}^{e x t}$ is $j$-th bar total internal force, corresponding to loads combination, resulting in the maximal (minimal) displacement $u_{t}$ magnitude; $\bar{S}_{t, j}$ is $j$-th bar internal force caused by unit force applied onto the node along the direction of nodal constrained displacement $u_{t}$ of the truss, being in the state prior to plastic collapse.

The structural member design stress versus buckling due EN3 [20] is calculated by:

$$
\sigma_{c r}=\chi \sigma_{y}
$$

where $\chi$ is certain bar reduction coefficient, depending on compressive member dimensionless slenderness $\bar{\lambda}$. The dimensionless slenderness is prescribed by ratio:

$$
\lambda=\lambda / \lambda_{E}
$$

Here the unit slenderness is defined by $\lambda=l_{b} / i$, where $l_{b}$ is an actual length of the buckled member, $i$ is the radius of gyration, $\lambda_{E}=\sqrt{\frac{\pi^{2} E}{\sigma_{y}}}$ is the Euler's slenderness. For example, the tube cross-sectional reduction coefficient analytically is described by the relation

$$
\chi=\frac{1}{\varphi+\sqrt{\varphi^{2}-\lambda^{2}}} \leq 1 \text {, where } \varphi=\frac{1}{2}\left[1+\alpha(\bar{\lambda}-0,2)+\bar{\lambda}^{2}\right],
$$

with $\alpha$ being the variance coefficient (eg $\alpha=0,21$ for hot laminated pipes, $\alpha=0,34$ for cold laminated pipes).

The truss-type structural members cross-sectional areas optimization problem is the non-linear mathematical programming problem, having one extremum. Two solutions cases due to possible admissible sets of variables are shown in Figs 2 and 3 in case of two optimized parameters. 


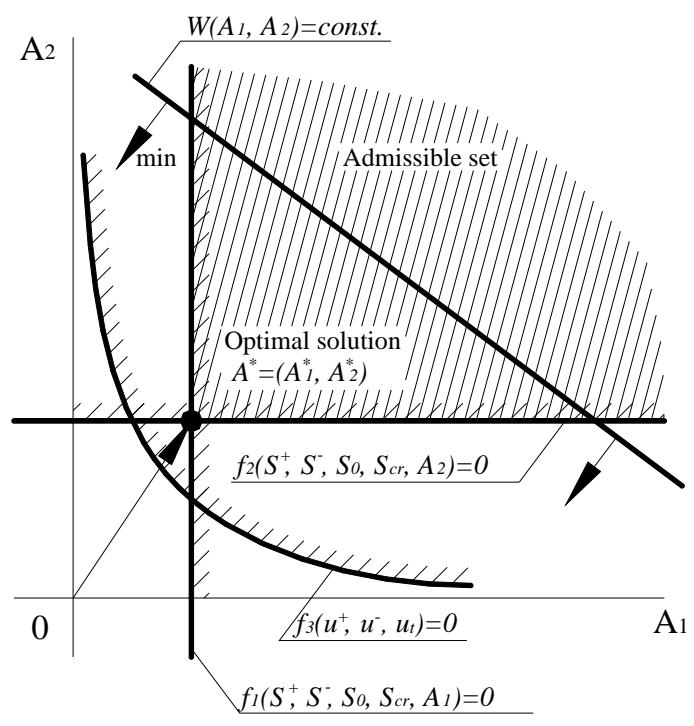

Fig 2. Piecewise-linear admissible set

The Fig 2 presents an optimal solution in the case when the displacement constraints are not activated. The optimization process is preconditioned by strength/stability constraints.

Fig 3 presents the case when the optimal solution is preconditioned also by stiffness constraints. In considered case the optimal solution contains the satisfied as equality leastwise one strength/stability condition in concert with stiffness constraint. It is obvious that the optimal solution objective function for the case results the more structural weight magnitude, when compared with the obtained one, presented in Fig 2.

Analysing the above Figures, one can obviously find that the optimization process converges to unique solution in both possible cases.

\subsection{Analysis and optimum design algorithm main steps}

The structure stochastic load combination problem in terms of created, constant per optimization process matrices/matrix, to predefine structural elastic response extreme values, is already provided. The minimum weight elastic-plastic truss-type structure optimization consists of the following eight steps:

1. Create the configuration matrices $[B]$ and $[R]$.

2. Determine the limit axial forces $\mathbf{S}_{0}$ and $\mathbf{S}_{c r}$. (Choose primary cross-sectional areas of bars for the start of optimization procedures, introduce the new ones, corresponding to the previous iteration optimization result, for the running optimization iteration).

3. Create structure discrete model quasidiagonal stiffness matrix for tensile-compressive elements $[\bar{K}]$ (Eqn (20)) and global stiffness matrix $[K]$.

4. Determine $\mathbf{S}_{e}^{+}, \mathbf{S}_{e}^{-}$and $\mathbf{u}_{e}^{+}, \mathbf{u}_{e}^{-}$(Eqns. (11) or (13)), applying the loading matrices or matrix.

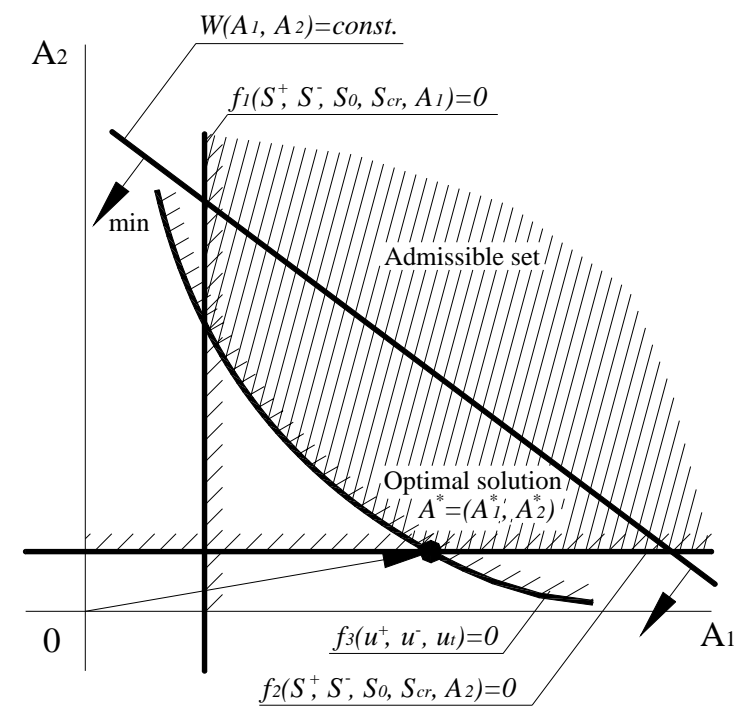

Fig 3. Non-linear admissible set

5. Create influence matrix of residual internal forces $[G]$ (Eqn (17)).

6. Solve the SSS evaluation quadratic programming problem to find residual structural response values (problem (14)).

7. Solve structure bars areas optimization problem under the presence of strength, stiffness and stability constraints (problem (25)).

8. Check the optimization problem prescribed convergence criterion in respect of previous iteration result. When it is not satisfied, repeat steps $2-8$. The prescribed criterion can be the fixed admitted tolerance of structure weight function, that of the bars areas (both criterions are correlated).

Note, that the number of iterations of optimization problem solution depends on successful starting point (ie chosen for 2-nd step primary input data (bars areas)). They can be chosen arbitrarily, solving eg the constant loading problem, or any similar one, being naturally close to actual constraints of the problem under investigation.

\subsection{Numerical example}

Consider the space steel structure of 25 bars (Fig 4), subjected to 3 different mutually independent stochastic load processes.

The first load $\tilde{F}_{1}(t)$ is applied to the 1-st node (direction cosines in respect of structure global axes $x, y, z$ are: $\cos \alpha_{x}=0,8146, \cos \alpha_{y}=0,2037$ and $\cos \alpha_{z}=0,5431$.

The second load $\tilde{F}_{2}(t)$ is applied to the 2-nd node (direction cosines in respect of structure global axes $x, y, z$ : $\cos \alpha_{x}=0,8304, \quad \cos \alpha_{y}=0,2491$ and $\cos \alpha_{z}=0,4983$. The third load $\tilde{F}_{3}(t)$ consists of simultaneous (fully correlated) action of two loads, being parallel to the axis $z$ 
and applied to the 3-rd and 6-th nodes, respectively. All three loads are prespecified by non-alternating direction processes (no direction changes). The load processes are described by stationary pulses (5). The load distribution function during activity periods satisfy the Gumbel's law:

$$
\hat{P}_{F}(F)=\exp (-\exp (a(F-u))),
$$

then

$$
\stackrel{\wedge}{P}^{-1} F(F)=u-\frac{1}{a}\left(\ln \left(-\ln P_{r}\right)\right) .
$$

The loads processes are described by the following parameters:

$$
\tilde{F}_{1}(t): \tau_{1}=91 \text { day, } u_{1}=141,62 \mathrm{kN}, a_{1}=1,90 \mathrm{kN},
$$

$$
q_{1}^{*}=0,2493, \quad p_{1}^{*}=0,7507
$$

$\tilde{F}_{2}(t): \tau_{2}=91$ day, $u_{2}=115,69 \mathrm{kN}, a_{2}=2,10 \mathrm{kN}$;

$$
q_{2}^{*}=0,1032, \quad p_{2}^{*}=0,8968
$$

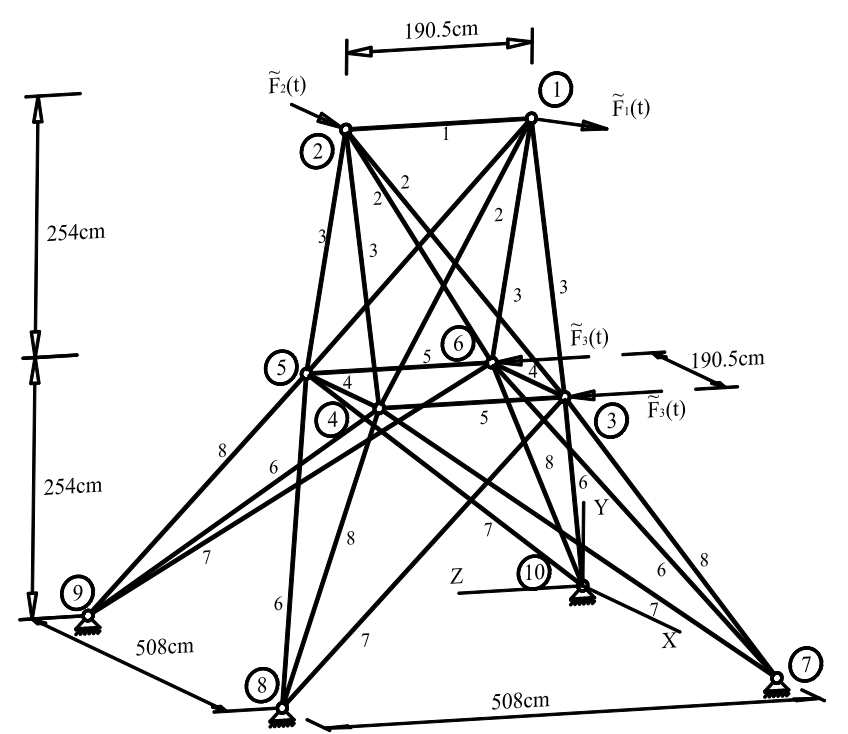

Fig 4. Truss design scheme

$$
\begin{gathered}
\tilde{F}_{3}(t): \quad \tau_{3}=0,26 \text { day, } u_{3}=19,82 \mathrm{kN}, \quad a_{3}=1,23 \mathrm{kN} ; \\
q_{3}^{*}=0,0039, \quad p_{3}^{*}=0,9961 .
\end{gathered}
$$

The truss service term is taken $T=50$ years, design probability $P_{r d}=P_{r s}=1-P_{r f}=0,999$.

Taking into account the problem features (direction nature of loads, design probability magnitude, service term, duration of pulses and probabilities of activity per pulses) the stochastic loading combinations (apexes) of polyhedron, describing the loads common action possible set, can be described by the following five combinations. Theses apexes of polyhedron, indicated by solid lines in Fig 5, represent the loads possible variation volume, created due to design probability $P_{r d}$. The dashed lines indicate the loads variation bounds in case of repeated-variable loading in terms of extreme loads variation bounds. The polyhedron apexes assembled into the loading matrix, read:

$$
[F]^{T}=\left[\begin{array}{ccc}
\max F_{1}(t) & 0 & 0 \\
T & & \\
\max F_{1}(t) & 0 & \max F_{3}(t) \\
T & & \tau 1 \\
0 & \max F_{2}(t) & 0 \\
& T & \\
0 & \max F_{2}(t) & \max F_{3}(t) \\
& T & \tau 2 \\
0 & 0 & \max F_{3}(t)
\end{array}\right] .
$$

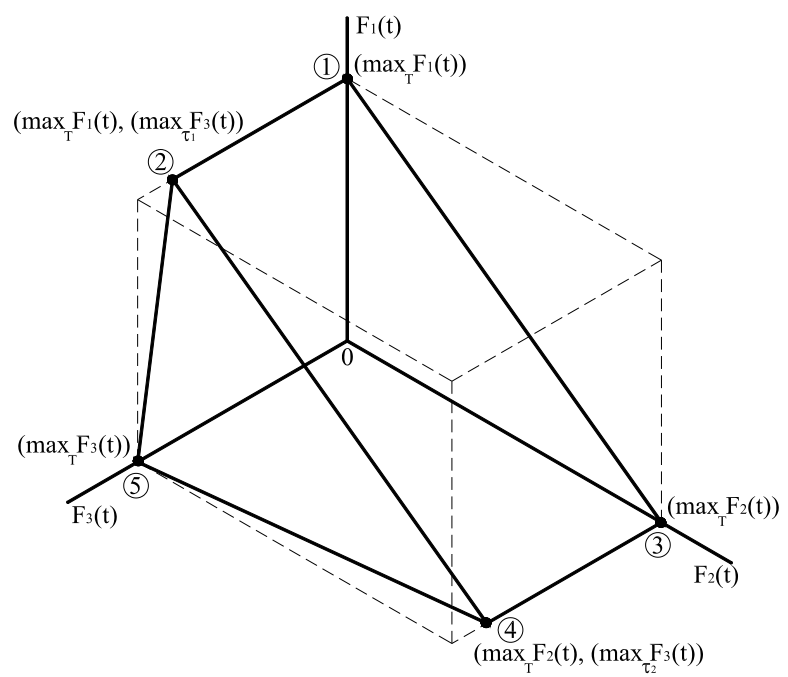

Fig 5. Loading process polyhedron

Taking into account loads constant directions, the fixed by (9) design probabilities are to be chosen as $P_{r d}=P_{r d(\max )}, P_{r d(\min )}=0$ and the other quintiles to be equal 0,5 (Eqn (8)). Then applying (11) and (12) to calculate determinate loads magnitudes, the loading matrix finally reads:

$$
[F]^{T}=\left[\begin{array}{ccc}
147,31 & 0 & 0 \\
147,31 & 0 & 20,10 \\
0 & 120,42 & 0 \\
0 & 120,42 & 20,10 \\
0 & 0 & 30,00
\end{array}\right] .
$$

Eight groups (ie 8 different truss areas) of pipe type cold laminated bars, (Fig 3) are optimized to ensure truss minimal weight under presence strength, stiffness, stability and constructional constraints. The material properties are: yield limit $\sigma_{y}=240 \mathrm{MPa}$, elasticity modulus $E=207 \mathrm{GPa}$. Truss nodes 1 and 2 extreme displacements are constrained in directions $x$ and $z$ by $1 \mathrm{~cm}$. The 
constructional requirement introduces minimal bars area to be $2 \mathrm{~cm}^{2}$.

The optimization process was realized per 13 iterations (see Table). As starting point input data (see row 0 of the Table) was taken an optimization solution of the considered truss, loaded by the same three loads assuming them to be applied as constant values with extreme magnitudes, presented in the above loading matrix, ie: $174,31 \mathrm{kN} ; 120,42 \mathrm{kN} ; 30,00 \mathrm{kN}$ for the first, second and third loads respectively. The starting point (optimal solution) was obtained in 12 iterations, applying the same algorithm, for its starting point taking all areas to be equal to $10 \mathrm{~cm}^{2}$ ones.

The stochastical loading process subjected truss optimal solution was reached in 13 iterations (the last row of the Table) with $0,017 \%$ tolerance for $3 \mathrm{rd}$ and 7 th areas and $0 \%$ tolerance for remaining areas and total weight of the structure.

Some notes on optimal truss behavior, being adapted to considered loading process. The 1 st node reached it allowable $1 \mathrm{~cm}$ extreme magnitude, while the other displacements do not achieve this magnitude. The 4 th and 5 th areas reached their minimum equal to $2 \mathrm{~cm}^{2}$ area magnitudes. The 1-2, 1-4, 4-9 truss members are loaded up to the critical values. Critical states for tensile members are not achieved. A deformable behaviour comparison of the optimized truss with the one, obtained for the constantly loaded truss (ie with the starting point representing optimization result - row 0 of the Table). The constantly loaded truss 1-2, 1-4 members were loaded up to the critical values. Critical states for tensile members were not achieved.

\section{Conclusions}

1. The performed numerical experiment illustrates the efficiency of proposed algorithm, when applying the proposed optimization under presence stiffness and sta- bility constraints method for the truss, subjected to stochastic loading process.

2. The convenient loading approximation method required for iterative structural optimization to identify extreme magnitudes of structural response with prescribed reliability per reference period of time is presented. The loading process approximation principles can be also applied for other structural analysis and design cases.

3. The new truss structural optimization model is presented. The result reliability is ensured by the application of the extreme energy principle for analysis problem to fix the actual structural stress and strain state in each optimization cycle.The proposed optimization method principles can be implemented for other types of structures.

4. The evaluation of stochastic loading process nature, implemented into structural optimization process, ensures material resources savings, when compared with the optimal result, obtained when representing the loading process either by repeated variable process or constant loads in terms of extreme magnitudes, achieved in the considered period of time.

\section{References}

1. Atrek, E.; Gallager, R. H.; Ragsdell, K. M. and Zienkiewicz, O. C. New directions in optimum structural design. New-York: John Wiley, 1984. 592 p.

2. Cyras, A. A. Mathematical models for the analysis and optimization of elastoplastic structures. Chichester: Ellis Horwood Lim., 1983. 121 p.

3. Levy, R. and Lev, O. E. Recent developments in structural optimization. J. Struct. Engng. ASCE 112, 1987, p. 19391962.

4. Cyras, A. and Norkus, A. On optimization of elastoplastic structures under random loading. Mech. Res. Comm., Vol 20, No 4, 1993, p. 293-299.

Optimization problem solutions per iterations

\begin{tabular}{c|c|c|c|c|c|c|c|c|c}
\hline $\begin{array}{c}\text { Iteration } \\
\text { number }\end{array}$ & $\begin{array}{c}\mathrm{A}_{1} \\
\left(\mathrm{~cm}^{2}\right)\end{array}$ & $\begin{array}{c}\mathrm{A}_{2} \\
\left(\mathrm{~cm}^{2}\right)\end{array}$ & $\begin{array}{c}\mathrm{A}_{3} \\
\left(\mathrm{~cm}^{2}\right)\end{array}$ & $\begin{array}{c}\mathrm{A}_{4} \\
\left(\mathrm{~cm}^{2}\right)\end{array}$ & $\begin{array}{c}\mathrm{A}_{5} \\
\left(\mathrm{~cm}^{2}\right)\end{array}$ & $\begin{array}{c}\mathrm{A}_{6} \\
\left(\mathrm{~cm}^{2}\right)\end{array}$ & $\begin{array}{c}\mathrm{A}_{7} \\
\left(\mathrm{~cm}^{2}\right)\end{array}$ & $\begin{array}{c}\mathrm{A}_{8} \\
\left(\mathrm{~cm}^{2}\right)\end{array}$ & $\begin{array}{c}\text { Truss weight } \\
(\mathrm{kg})\end{array}$ \\
\hline $\mathbf{0}$ & $\mathbf{2 , 0 0}$ & $\mathbf{8 , 0 8 9}$ & $\mathbf{1 2 , 5 4 0}$ & $\mathbf{2 , 0 0 0}$ & $\mathbf{4 , 1 6 3}$ & $\mathbf{5 , 3 8 3}$ & $\mathbf{2 , 7 5 9}$ & $\mathbf{1 7 , 8 1 7}$ & $\mathbf{5 1 9 , 3 5}$ \\
\hline 1 & 2,826 & 7,434 & 13,414 & 2,000 & 2,000 & 5,418 & 6,513 & 15,869 & 546,80 \\
\hline 2 & 3,565 & 7,791 & 11,726 & 2,000 & 2,000 & 2,767 & 5,515 & 10,920 & 435,63 \\
\hline 3 & 3,373 & 7,642 & 11,543 & 2,000 & 2,000 & 2,696 & 5,636 & 11,072 & 432,79 \\
\hline 4 & 3,737 & 7,760 & 11,507 & 2,000 & 2,000 & 2,682 & 5,604 & 10,990 & 432,74 \\
\hline 5 & 3,106 & 7,658 & 11,519 & 2,000 & 2,000 & 2,694 & 5,634 & 11,057 & 432,15 \\
\hline 6 & 3,686 & 7,780 & 11,495 & 2,000 & 2,000 & 2,681 & 5,603 & 10,988 & 432,71 \\
\hline 7 & 3,183 & 7,670 & 11,511 & 2,000 & 2,000 & 2,695 & 5,634 & 11,061 & 432,37 \\
\hline 8 & 3,702 & 7,760 & 11,500 & 2,000 & 2,000 & 2,684 & 5,608 & 11,000 & 432,78 \\
\hline 9 & 3,160 & 7,682 & 11,562 & 2,000 & 2,000 & 2,703 & 5,732 & 11,091 & 434,74 \\
\hline 10 & 3,696 & 7,732 & 11,573 & 2,000 & 2,000 & 2,696 & 5,746 & 11,020 & 435,51 \\
\hline 11 & 3,432 & 7,701 & 11,582 & 2,000 & 2,000 & 2,697 & 5,762 & 11,010 & 435,00 \\
\hline 12 & 3,429 & 7,702 & 11,576 & 2,000 & 2,000 & 2,697 & 5,764 & 11,010 & 435,00 \\
\hline $\mathbf{1 3}$ & $\mathbf{3 , 4 2 9}$ & $\mathbf{7 , 7 0 2}$ & $\mathbf{1 1 , 5 7 8}$ & $\mathbf{2 , 0 0 0}$ & $\mathbf{2 , 0 0 0}$ & $\mathbf{2 , 6 9 7}$ & $\mathbf{5 , 7 6 5}$ & $\mathbf{1 1 , 0 1 0}$ & $\mathbf{4 3 5 , 0 0}$ \\
\hline
\end{tabular}


5. Macke, M. A reliability-based design format for fatiguesensitive steel structures. In: Proc. of 8th Int. Conf. on Applic. on Statist. and Probab., Sidney, Australia, 1999, Roterdam: Balkema, 2000.

6. Sobzyk, K. Spenser, F. Random fatigue: from data to theory. Boston: Academic Press, 1992. 254 p.

7. Simoes, L. M. Stochastically dominant modes of frames by mathematical programming. J. Struct. Engng. ASCE 116, 1990, p. 1040-1060.

8. Ellis, J. H.; Zimmerman, J. J.; Corotis, R. B. Stochastic programs for identifying critical structures collapse mechanisms. Appl. Math. Modelling, Vol 15, No 7, 1991, p. 367373.

9. Khan, M. R. Optimality criterion techniques applied to frames having general cross-sectional relationships. AIAA J. 22(5), 1984, p. 669-676.

10. Jarmolajeva, E.; Atkočiūnas, J. Shakedown loading optimization under residual displacements - formulation and solution for circular plates. J. of Civ. Engng. and Manag., Vol VIII, No 1. Vilnius: Technika, 2002, p. 54-67.

11. Kalanta, S. New formulations of optimization problems of elasto-plastic bar structures under displacement constraints. Mechanics (Mechanika), 5(20). Kaunas: Technologija, 1999, p. 9-16 (in Russian).

12. Karkauskas, R. and Norkus, A. Optimization of geometrically nonlinear elastic-plastic structures under stiffness constraints. Mech. Res. Comm., Vol 28, No 5, 2001, p. 505512.
13. Bucher, C. Some recent software developments for stochastic structural analysis. In: Proc. of 7th Int. Conf. on Structur. Safety and Reliability (ICOSSAR), Kioto, Japan, 1997, Roterdam: Balkema, 1981.

14. Ditlevsen, O. and Madsen, H. O. Probabilistic modelling of man-made load processes and their individual and combined effects. In: Proc. of 3rd Int. Conf. on Struct. Safety and Reliability (ICOSSAR), Kioto, Japan, 1997, Roterdam: Balkema, 1981.

15. Mori Yasuhiro, Kato Takahiro. Probabilistic models of combinations of stochastic loads for limit state design. J. Struct. Safety 25, 2003, p. 69-97.

16. Larrabee, R. D. Approximate stochastic analysis of combined loading. Research report R78-28 of Dept. of Civ. Engng., 1978.

17. Madsen, H. O. On stochastic load combination. Report No 30, Solid Mech. Div. Univ. of Waterloo, Ontario, Canada, 1978.

18. Karkauskas, R. Analysis of geometrically nonlinear elastoplastic structures. Civil Engineering (Statyba), 4 (1). Vilnius: Technika, 1998, p. 36-42.

19. Atkočiūnas, J. Design of elastoplastic systems under repeated loading (Расчет упругопластических систем при повторных нагружениях). Vilnius: Mokslo ir enciklopediju leidykla, 1994. 146 p. (in Russian).

20. EC3. Eurocode No 3. Design of Steel Structures, Vol 1, Nov 1990, Vol 2, July 1990. 